ARTIGO ORIGINAL ORIGINAL ARTICLE

\title{
Como promover a desospitalização devido ao transplante de órgãos sólidos? Panorama atual no Brasil e profilaxia da infecção pelo citomegalovírus com valganciclovir
}

\author{
How to promote deinstitutionalization due to solid \\ organ transplantation? Current landscape in Brazil and \\ cytomegalovirus infection prophylaxis with valganciclovir \\ Camila Rufino ${ }^{1}$, Daniela Carlini', Marcia Alves ${ }^{1}$, Hellen Soo Jin Kim²
}

DOI: 10.21115/JBES.v8.n1.p47-57

\begin{abstract}
Palavras-chave:
valganciclovir, ganciclovir, citomegalovírus, transplante, alta do paciente
\end{abstract}

\section{Keywords:}

valganciclovir, ganciclovir, cytomegalovirus, transplant, patient discharge

\section{RESUMO}

Objetivo: $O$ estudo teve o objetivo de avaliar o panorama dos transplantes de órgãos sólidos (TOS) no Brasil e compreender as diferenças entre a profilaxia oral com valganciclovir e o tratamento preemptivo intravenoso com ganciclovir em pacientes de alto risco para desenvolvimento da doença pelo citomegalovírus (CMV) (D+/R-), e seu potencial impacto na desospitalização. Métodos: Por meio do Sistema de Informações Hospitalares do SUS (SIH/SUS), uma análise retrospectiva foi realizada para avaliar os principais centros de transplantes brasileiros e obter os TOS realizados em 2014. Os cálculos foram baseados na permanência hospitalar média por TOS, tempo de internação para cada abordagem farmacológica e número de pacientes D+/R-. A permanência hospitalar para tratamento preemptivo e profilaxia foram baseadas em diretrizes de uma instituição brasileira de referência. A taxa de infecção de CMV foi obtida de uma revisão da literatura. O desenvolvimento da doença pelo CMV após tratamento preemptivo ou profilaxia não foi considerado. Resultados: Em 2014, os centros de transplante avaliados realizaram um total de 6.912 TOS. O valganciclovir profilático proporcionou anualmente 21 dias a menos de hospitalização por paciente. A partir da relação do valor incremental (21 dias) entre a utilização de ganciclovir e valganciclovir e a média geral de permanência hospitalar no SUS para qualquer procedimento (5,6 dias), sugere-se que 3,75 novas internações por qualquer causa poderiam ocorrer por cada paciente em profilaxia. Conclusão: $O$ valganciclovir profilático para pacientes $D+/ R$ - submetidos a TOS é potencialmente capaz de promover a desospitalização, permitindo maior comodidade ao paciente transplantado e utilização racional de recursos pela instituição.

\begin{abstract}
Objective: The study aimed to assess the landscape of solid organ transplants (SOT) in Brazil and understand differences between oral prophylaxis with valganciclovir and intravenous preemptive treatment with ganciclovir in patients at high risk for developing disease by cytomegalovirus (D+ /R-), and their potential impact on deinstitutionalization. Methods: Based on the Brazilian Hospital Information System (SIH/SUS) database, a retrospective analysis was performed to assess the main Brazilian transplantation centers and obtain the SOT performed in 2014. The analysis was based on time of hospitalization according to SOT, LOS (Length of Stay) of each pharmacological approach and number of D+/R- patients. LOS for preemptive treatment and prophylaxis was taken from a Brazilian reference institution guideline. The CMV infection rate was obtained from a literature
\end{abstract}

Recebido em: 24/02/2016. Aprovado para publicação em: 22/03/2016

'Hoffmann-La Roche Ltd, São Paulo, Brazil

'Sense Company, São Paulo, Brazil.

Instituição onde o trabalho foi executado: Hoffmann-La Roche Ltd, São Paulo, SP, Brasil.

Informações sobre auxílios recebidos sob a forma de financiamento, equipamentos ou medicamentos: o estudo foi desenvolvido por colaboradores Hoffmann-La Roche Ltd, Brasil.

Conflitos de interesse: Hoffmann-La Roche Ltd é a detentora dos direitos de comercialização e promoção de valganciclovir no Brasil. O desenvolvimento deste manuscrito foi feito por Hoffmann-La Roche Ltd sob colaboração da Sense Company.

Congressos: o resumo do estudo foi submetido no 21st Annual International Meeting, organizado pela International Society for Pharmacoeconomics and Outcomes Research.

Autor Correspondente: Camila Rufino. Av. Engenheiro Billings, 1729 - Jaguaré, São Paulo/SP - 05321-900. E-mail: camila.rufino@ roche.com 
review. Development of CMV disease after both prophylaxis and preemptive treatment was not considered in the analysis. Results: In 2014, the transplant centers performed a total of 6,912 SOT. The valganciclovir prophylaxis resulted in reduction of 21 days of LOS per patient. Based on the ratio of incremental daily between the use of ganciclovir and valganciclovir (21 days) and overall mean hospital stay in the SUS to any procedure (5.6 days), it may be suggested that 3.75 new admissions for any cause could occur in addition, for each patient treated with prophylactic valganciclovir. Conclusion: Valganciclovir prophylaxis for patients D+ /R- undergoing TOS is potentially able to promote deinstitutionalization, allowing greater convenience to patients transplanted and promoting the rational use of resources by the institution.

\section{Introdução}

O Brasil representa o segundo país do mundo em número de transplantes e possui um programa público de saúde respeitado globalmente voltado para esta finalidade. Apesar disso, ainda existem filas de espera e problemas relatados pelo sistema, entre eles a taxa de doadores insuficiente por não autorização familiar, parada cardíaca inviabilizando a doação do órgão ou contraindicação médica, carência de equipes especializadas e também a falta de estrutura e leitos hospitalares (Associação Brasileira de Transplante de Órgãos (ABTO), 2014; Marinho, 2006).

A desospitalização, ou redução do tempo de permanência hospitalar, é uma tendência mundial em um cenário cujos recursos e leitos hospitalares são cada dia mais limitados. Seu objetivo é evitar a longa e, algumas vezes, desnecessária permanência do paciente em ambiente hospitalar, o que impacta em questões clínicas - como aumento do número de infecções hospitalares - e em questões econômicas. Além disso, a ocupação de leitos por longos períodos resulta em impossibilidade de utilização dos mesmos por outros pacientes que possam necessitar, levando a um congestionamento no sistema. Desta forma, medidas como o home care, instituições e centros de reabilitação, e substituição de tratamentos venosos por orais podem contribuir para esta gestão mais eficiente dos sistemas de saúde (Bordonaro et al., 2012).

Entre os fatores que influenciam no tempo de permanência hospitalar nos pacientes submetidos a transplante de órgãos sólidos (TOS) encontra-se a necessidade de profilaxia contra o citomegalovírus (CMV), um vírus ubíquo da família herpes vírus (HHV5) que pode ser transmitido pela transfusão de sangue, saliva, urina, transferência placentária, aleitamento materno e contato sexual, como também por transplante de células-tronco hematopoiéticas ou de órgãos sólidos (Alexopoulos et al., 2012; Giakoustidis et al., 2012). Sem a profilaxia adequada, aproximadamente $80 \%$ dos receptores de TOS podem desenvolver infecção pelo CMV (Junqueira et al., 2008; Roman et al., 2014).
A imunossupressão, necessária no momento do transplante para prevenir a rejeição aguda do órgão, coloca o receptor em alto risco de desenvolvimento da doença causada pelo CMV (Alexopoulos et al., 2012). Os principais fatores de risco que influenciam na ocorrência da doença após o TOS incluem: o estado sorológico do receptor e do doador e o nível de imunossupressão. Os pacientes receptores soronegativos-CMV, cujos doadores são soropositivos-CMV com infecção primária (D+/R-), possuem maiores taxas de doença sintomática e disseminada, além de um maior risco de rejeição do enxerto, infecções secundárias, aterosclerose e menor sobrevida (Harvala et al., 2013; Razonable, 2010).

A soroprevalência do CMV entre a população geral varia de 30-97\%, aumentando com a idade (Giakoustidis et al., 2012). Em pacientes imunocompetentes, a infecção primária de CMV pode ser assintomática, enquanto em pacientes com a função imunológica comprometida, o CMV pode ocasionar complicações clínicas mais graves e, em alguns casos, fatais (Junqueira et al., 2008; Razonable, 2010).

A combinação D+/R-corresponde a $20 \%$ de todos os órgãos transplantados e há um risco de 50 a 70\% para o desenvolvimento da doença causada pelo CMV. Já as combinações $\mathrm{D}+/ \mathrm{R}+$ e D-/R+ somam 70\% de todos os órgãos transplantados, com 10 a 20\% de risco de desenvolver a doença (Junqueira et al., 2008).

Podem ser observados efeitos diretos e indiretos causados por essa doença, que se manifestam clinicamente em qualquer órgão. Os efeitos diretos referem-se à própria infecção caracterizada como uma síndrome viral ou doença invasiva, que pode afetar o trato gastrointestinal, pulmão, fígado e rins. Já os efeitos indiretos podem ser vistos em pacientes que apresentam viremia pelo CMV, mesmo sem manifestação clínica, como o aumento de coinfecção com outros agentes infecciosos, maior incidência de rejeições, perda do enxerto e morte (Hospital Israelita Albert Einstein, 2011; Razonable, 2010; Roman et al., 2014).

Atualmente, existem duas estratégias aceitas para a prevenção e o manejo da doença pelo CMV: profilaxia antiviral e tratamento preemptivo. Na profilaxia, os pacientes recebem um tratamento antiviral para prevenir o desenvolvimento da infecção pelo CMV (Alexopoulos et al., 2012). Já na terapia preemptiva, os pacientes são monitorados para a evidência de sinais de replicação do CMV através de ensaios sensíveis, geralmente utilizando testes quantitativos de amplificação de ácidos nucleicos por reação em cadeia da polimerase (PCR) e detecção da replicação do CMV em infecções assintomáticas. A terapia antiviral é administrada para evitar a progressão da doença clínica sintomática (Alexopoulos et al., 2012; Bruminhent \& Razonable, 2014).

De acordo com o Protocolo de Diagnóstico e Tratamento de infecção pelo CMV em transplantados de órgãos sólidos do Hospital Albert Einstein (Hospital Israelita Albert Einstein, 
2012), o ganciclovir (GCV) e o valganciclovir (VGCV) são as opções antivirais mais adequadas respectivamente para tratamento preemptivo e profilaxia do CMV.

O VGCV é um pró-fármaco do GCV, indicado para a profilaxia da doença causada pelo CMV em receptores de transplante de órgãos sólidos de alto risco (D+/R-), de administração oral (Roche, 2014), sem necessidade de hospitalização durante sua administração.

Esse estudo teve como objetivo avaliar o panorama dos TOS no Brasil, incluindo sua distribuição geográfica, classificação por tipo de órgão e duração média das hospitalizações e compreender as diferenças entre a profilaxia oral com VGCV e o tratamento preemptivo intravenoso com GCV em pacientes de alto risco ( $D+/ R-$ ) e seu potencial impacto na desospitalização.

\section{Métodos}

Uma análise retrospectiva foi realizada por meio do Sistema de Informações Hospitalares do SUS (SIH/SUS) para avaliar os principais centros de transplantes brasileiros em 2014 (Ministério da Saúde, 2016a). As informações obtidas foram classificadas e agrupadas de acordo com o órgão sólido transplantado e a instituição hospitalar.

O tempo médio de hospitalização para todos os TOS foi calculado a partir de uma média ponderada, baseada na quantidade de TOS realizados em 2014 (SIH/SUS) e seus respectivos valores de média de permanência hospitalar (específicos para cada órgão sólido), obtidos pelo Sistema de Gerenciamento da Tabela de Procedimentos, Medicamentos e Órteses/Próteses e Materiais Especiais do SUS (SIGTAP/SUS) (Ministério da Saúde, 2016b).

O tempo de permanência hospitalar específico para o tratamento preemptivo foi definido de acordo com as Diretrizes Assistenciais do Hospital Albert Einstein, que preconiza o total de 21 dias (Hospital Israelita Albert Einstein, 2011). O tempo de hospitalização para profilaxia com VGCV foi estimado como zero, assumindo-se a premissa de que o paciente tratado com VGCV não necessita de internação por sua administração oral (Roche, 2014). O desenvolvimento da doença pelo CMV após a profilaxia ou o tratamento preemptivo e seu respectivo tempo de hospitalização não foram considerados na análise.

Além dos panoramas com valores totais, demais cálculos foram realizados sob duas perspectivas: paciente e instituição hospitalar.
Sob a perspectiva do paciente, o cálculo de diárias de hospitalização para ambas abordagens (profilaxia e tratamento preemptivo) foi realizado por meio da comparação da soma do intervalo de tempo de internação entre VGCV profilático e GCV em tratamento preemptivo. Os resultados foram expressos em dias de hospitalização evitados por paciente tratado com VGCV e potenciais utilizações dos leitos desocupados por outros pacientes, para quaisquer finalidades terapêuticas. Para tal análise, ainda foi consultado o SIH/ SUS, acerca da média de permanência para todos os procedimentos hospitalares disponíveis (cirúrgicos ou não cirúrgicos, em todas as especialidades médicas).

A população de pacientes de alto risco (D+/R-) foi calculada a partir da prevalência da infecção por CMV no Brasil $(96,4 \%)$, obtida em um estudo brasileiro. A partir desse dado, assumiu-se que todos os doadores seriam soropositivos-CMV (D+), e que 3,6\% dos pacientes não apresentariam a infecção, ou seja, soronegativos-CMV (R-) (Souza et al., 2010).

Sob a perspectiva da instituição, o cálculo populacional foi realizado apenas para os centros de transplante que apresentaram valores de populações D+/R- maiores que 1 . O cálculo foi elaborado por meio da multiplicação dos resultados por paciente pelo número de pacientes D+/R- por instituição.

\section{Resultados}

A partir do SIH/SUS, foram avaliados 122 centros de transplante brasileiros. No total, 6.912 TOS foram realizados no ano de 2014, com destaque para os transplantes de rim ( $n=4.931)$ e fígado ( $n=1.538$ ), que apresentaram os maiores números de procedimentos (Tabela 1). As maiores concentrações de centros de transplante estão localizadas nas regiões Sudeste e Sul do Brasil. As instituições brasileiras que apresentaram maiores quantidades de TOS foram a Irmandade Santa Casa de Misericórdia de Porto Alegre ( $n=327$ transplantes), Hospital das Clínicas da FMUSP ( $n=397$ transplantes) e Hospital do Rim e Hipertensão ( $n=687$ transplantes). As distribuições dos transplantes realizados por centro, por órgão e classificados de acordo com a região geográfica, estão apresentadas nas Tabelas 2 a 6 .

Desconsiderando-se o tipo de abordagem farmacológica voltada para a prevenção da infecção por CMV (tratamento preemptivo e profilaxia), o tempo de internação médio para todos os órgãos sólidos transplantados foi de 11 dias, com base na média de permanência hospitalar obtida pelo SIG-

Tabela 1. Número total de transplantes de órgãos sólidos realizados. Brasil, SIH/SUS, 2014. (Ministério da Saúde, 2016a)

\begin{tabular}{llllllll}
\hline & Coração & Fígado & Pâncreas & Pâncreas/rim & Pulmão & Rim \\
\hline TOTAL & 263 & 1.538 & 20 & 102 & 58 & 4.931 & \\
\hline
\end{tabular}

SIH/SUS: Sistema de Informações Hospitalares do SUS. 
Tabela 2. Relação de instituições e TOS realizados. Brasil, região Norte, SIH/SUS, 2014. (Ministério da Saúde, 2016a)

\begin{tabular}{|c|c|c|c|c|c|c|c|c|}
\hline UF & Instituição & Coração & Fígado & Pâncreas & Pâncreas/Rim & Pulmão & Rim & TOTAL \\
\hline PA & Hospital Ophir Loyola & - & - & - & - & - & 25 & 25 \\
\hline AM & Hospital Santa Julia & - & - & - & - & - & 23 & 23 \\
\hline$A C$ & Fundação Hospital do Acre & - & 3 & - & - & - & 9 & 12 \\
\hline PA & Hospital Regional Público do Araguaia & - & - & - & - & - & 11 & 11 \\
\hline RO & Hospital de base Porto Velho & - & - & - & - & - & 6 & 6 \\
\hline AM & Fundação Hospital Adriano Jorge & - & 3 & - & - & - & - & 3 \\
\hline Total & & 0 & 6 & 0 & 0 & 0 & 74 & 80 \\
\hline
\end{tabular}

AC: Acre; AM: Amazonas; PA: Pará; RO: Rondônia; UF: Unidade da Federação.

Tabela 3. Relação de instituições e TOS realizados. Brasil, região Nordeste, SIH/SUS, 2014. (Ministério da Saúde, 2016a)

\begin{tabular}{|c|c|c|c|c|c|c|c|c|}
\hline UF & Instituição & Coração & Fígado & Pâncreas & Pâncreas/Rim & Pulmão & $\operatorname{Rim}$ & TOTAL \\
\hline$P E$ & IMIP & 17 & 35 & - & 4 & - & 175 & 231 \\
\hline CE & Hospital Universitário Walter Cantidio & - & 131 & - & - & - & 93 & 224 \\
\hline CE & Hospital Geral de Fortaleza & - & 44 & - & 5 & - & 153 & 202 \\
\hline$P E$ & Real Hospital Português & 9 & & - & - & - & 83 & 92 \\
\hline $\mathrm{PE}$ & Hospital Jayme da Fonte & - & 72 & - & - & - & & 72 \\
\hline RN & Hospital Universitário Onofre Lopes & - & - & - & - & - & 67 & 67 \\
\hline BA & Hospital Ana Nery & - & - & - & - & - & 52 & 52 \\
\hline BA & Hospital Português & - & 36 & - & - & - & 3 & 39 \\
\hline PB & Hospital Antonio Targino & - & - & - & - & - & 36 & 36 \\
\hline CE & $\begin{array}{l}\text { Hospital de Messejana Dr Carlos } \\
\text { Alberto Studart Gomes }\end{array}$ & 19 & - & - & - & 8 & - & 27 \\
\hline MA & Hospital Universitário HUUFMA & - & - & - & - & - & 25 & 25 \\
\hline BA & Hospital São Rafael & - & 18 & - & - & - & 5 & 23 \\
\hline $\mathrm{PE}$ & Casa de Saúde Santa Efigênia Ltda & - & - & - & - & - & 21 & 21 \\
\hline BA & Hospital Calixto Midlej Filho & - & - & - & - & - & 20 & 20 \\
\hline $\mathrm{PI}$ & Casamater & - & - & - & - & - & 18 & 18 \\
\hline $\mathrm{Pl}$ & Hospital Getúlio Vargas & - & - & - & - & - & 14 & 14 \\
\hline CE & Hospital São Carlos & - & 12 & - & - & - & & 12 \\
\hline CE & Hospital São Raimundo & - & - & - & - & - & 12 & 12 \\
\hline $\mathrm{AL}$ & Chama & - & - & - & - & - & 10 & 10 \\
\hline $\mathrm{AL}$ & Santa Casa De Misericórdia de Maceió & 3 & - & - & - & - & 4 & 7 \\
\hline $\mathrm{PE}$ & Hospital das Clinicas & - & - & - & - & - & 6 & 6 \\
\hline MA & Incor Santa Mônica & - & - & - & - & - & 4 & 4 \\
\hline $\mathrm{AL}$ & Hospital do Açúcar & - & - & - & - & - & 3 & 3 \\
\hline RN & Hospital do Coração de Natal & - & 2 & - & - & - & & 2 \\
\hline BA & Hospital Espanhol & - & - & - & - & - & 1 & 1 \\
\hline PB & Hospital Unimed João Pessoa & - & 1 & - & - & - & & 1 \\
\hline \multicolumn{2}{|l|}{ Total } & 48 & 351 & 0 & 9 & 8 & 805 & 1221 \\
\hline
\end{tabular}

AL: Alagoas; BA: Bahia; CE: Ceará; IMIP: Instituto de Medicina Integral Professor Fernando Figueira; MA: Maranhão;

PB: Paraíba; PE: Pernambuco; PI: Piauí; RN: Rio Grande do Norte; UF: Unidade da Federação. 
Tabela 4. Relação de instituições e TOS realizados. Brasil, região Centro-Oeste, SIH/SUS, 2014. (Ministério da Saúde, 2016a)

\begin{tabular}{|c|c|c|c|c|c|c|c|c|}
\hline UF & Instituição & Coração & Fígado & Pâncreas & Pâncreas/Rim & Pulmão & $\operatorname{Rim}$ & TOTAL \\
\hline DF & $\begin{array}{l}\text { Instituto de Cardiologia } \\
\text { do Distrito Federal }\end{array}$ & 20 & 48 & - & - & - & 36 & 104 \\
\hline $\mathrm{GO}$ & Santa Casa de Misericórdia De Goiânia & - & - & - & - & - & 39 & 39 \\
\hline DF & $\begin{array}{l}\text { Hospital De Base Do Distrito } \\
\text { Federal - HBDF }\end{array}$ & - & - & - & - & - & 38 & 38 \\
\hline DF & Hospital Universitário De Brasília & - & - & - & - & - & 27 & 27 \\
\hline GO & Hospital Santa Genoveva & - & - & - & 3 & - & 22 & 25 \\
\hline MS & Santa Casa & - & - & - & - & - & 3 & 3 \\
\hline Total & & 20 & 48 & 0 & 3 & 0 & 165 & 236 \\
\hline
\end{tabular}

DF: Distrito Federal; GO: Goiás; MS: Mato Grosso do Sul; UF: Unidade da Federação.

Tabela 5. Relação de instituições e TOS realizados. Brasil, região Sudeste, SIH/SUS, 2014. (Ministério da Saúde, 2016a)

\begin{tabular}{|c|c|c|c|c|c|c|c|c|}
\hline UF & Instituição & Coração & Fígado & Pâncreas & Pâncreas/Rim & Pulmão & $\operatorname{Rim}$ & TOTAL \\
\hline SP & Hospital do Rim e Hipertensão & - & - & 2 & 22 & - & 663 & 687 \\
\hline SP & Hospital das Clinicas São Paulo & - & 135 & 1 & 7 & - & 254 & 397 \\
\hline RJ & Hospital São Francisco De Assis & - & 62 & - & - & - & 206 & 268 \\
\hline SP & Hospital Albert Einstein & 9 & 97 & - & 1 & 4 & 75 & 186 \\
\hline SP & $\begin{array}{l}\text { Hospital das Clinicas da } \\
\text { UNICAMP de Campinas }\end{array}$ & 3 & 44 & - & - & - & 119 & 166 \\
\hline MG & Hospital Felicio Rocho & 8 & 29 & 1 & 14 & - & 100 & 152 \\
\hline SP & Hospital Bandeirantes & - & 56 & 12 & 13 & - & 56 & 137 \\
\hline SP & Hospital das Clinicas de Botucatu & - & - & - & - & - & 124 & 124 \\
\hline SP & $\begin{array}{l}\text { Hospital de Base de São } \\
\text { José do Rio Preto }\end{array}$ & - & 36 & - & 3 & - & 75 & 114 \\
\hline MG & Hospital das Clinicas da UFMG & 29 & 47 & - & 2 & 1 & 13 & 92 \\
\hline SP & $\begin{array}{l}\text { Hospital de Transplante } \\
\text { Euryclides de Jesus Zerbini }\end{array}$ & - & 33 & - & - & - & 59 & 92 \\
\hline MG & Santa Casa de Belo Horizonte & - & - & - & 1 & - & 89 & 90 \\
\hline ES & Hospital Meridional & 7 & 40 & - & - & - & 41 & 88 \\
\hline SP & $\begin{array}{l}\text { HC da FMUSP Instituto do } \\
\text { Coração Incor São Paulo }\end{array}$ & 60 & - & - & - & 24 & 0 & 84 \\
\hline SP & $\begin{array}{l}\text { Hospital São Joaquim } \\
\text { Beneficência Portuguesa }\end{array}$ & 4 & 17 & - & 1 & - & 60 & 82 \\
\hline MG & $\begin{array}{l}\text { Santa Casa de Misericórdia } \\
\text { de Juiz de Fora }\end{array}$ & - & - & - & - & - & 79 & 79 \\
\hline MG & Hospital Universitário São José & - & - & - & - & - & 77 & 77 \\
\hline SP & $\begin{array}{l}\text { Hospital das Clinicas } \\
\text { FAEPA Ribeirão Preto }\end{array}$ & - & 28 & - & 2 & - & 45 & 75 \\
\hline RJ & Hospital Adventista Silvestre & - & 53 & - & - & - & 20 & 73 \\
\hline MG & Hospital Santa Casa de Montes Claros & - & 17 & - & - & - & 48 & 65 \\
\hline RJ & Hospital Geral De Bonsucesso & - & - & - & - & - & 59 & 59 \\
\hline SP & $\begin{array}{l}\text { Instituto Dante Pazzanese De } \\
\text { Cardiologia São Paulo }\end{array}$ & 20 & - & - & - & - & 30 & 50 \\
\hline ES & Hospital Evangélico De Vila Velha & - & - & - & - & - & 47 & 47 \\
\hline MG & Hospital Marcio Cunha & - & - & - & - & - & 39 & 39 \\
\hline MG & Hospital Evangélico & - & - & - & - & - & 34 & 34 \\
\hline
\end{tabular}




\begin{tabular}{|c|c|c|c|c|c|c|c|c|}
\hline SP & $\begin{array}{l}\text { Santa Casa De Misericórdia } \\
\text { De São Jose Dos Campos }\end{array}$ & - & 34 & - & - & - & & 34 \\
\hline SP & $\begin{array}{l}\text { Santa Casa De São Paulo } \\
\text { Hospital Central São Paulo }\end{array}$ & - & 7 & - & - & - & 26 & 33 \\
\hline MG & $\begin{array}{l}\text { Hospital das Clinicas Samuel } \\
\text { Libanio Pouso Alegre }\end{array}$ & - & - & - & - & - & 32 & 32 \\
\hline SP & Hospital Samaritano & - & - & - & - & - & 32 & 32 \\
\hline SP & $\begin{array}{l}\text { Hospital do câncer Antônio } \\
\text { Cândido Camargo }\end{array}$ & - & 32 & - & - & - & - & 32 \\
\hline SP & Hospital Santa Marcelina São Paulo & - & - & - & - & - & 30 & 30 \\
\hline SP & $\begin{array}{l}\text { Hospital São Paulo Hospital } \\
\text { de Ensino da UNIFESP }\end{array}$ & 1 & 23 & - & - & - & 3 & 27 \\
\hline MG & Hospital Santa Catarina & - & - & - & - & - & 26 & 26 \\
\hline SP & Santa Casa de Marilia & - & - & - & - & - & 22 & 22 \\
\hline MG & Hospital De Clinicas De Uberlândia & - & - & - & - & - & 19 & 19 \\
\hline RJ & Hospital Universitário Pedro Ernesto & - & - & - & - & - & 19 & 19 \\
\hline RJ & Hospital Universitário Antonio Pedro & - & - & - & - & - & 18 & 18 \\
\hline SP & Hospital Santa Lucinda Sorocaba & - & - & - & - & - & 16 & 16 \\
\hline MG & Hospital da Baleia & - & - & - & - & - & 14 & 14 \\
\hline SP & Santa Casa de Ribeirão Preto & - & - & - & - & - & 14 & 14 \\
\hline RJ & $\begin{array}{l}\text { Hospital Universitário } \\
\text { Clementino Fraga Filho }\end{array}$ & - & 13 & - & - & - & - & 13 \\
\hline RJ & $\begin{array}{l}\text { Hospital Estadual Transplante } \\
\text { câncer e cirurgia infantil }\end{array}$ & - & 6 & - & - & - & 6 & 12 \\
\hline SP & $\begin{array}{l}\text { Santa Casa Hospital Dr } \\
\text { Aristoteles Oliveira Martins }\end{array}$ & - & - & - & - & - & 7 & 7 \\
\hline MG & Hospital São Francisco De Assis & - & - & - & - & - & 6 & 6 \\
\hline RJ & Instituto Nacional de Cardiologia & 6 & - & - & - & - & - & 6 \\
\hline MG & Hospital São João de Deus & - & - & - & - & - & 5 & 5 \\
\hline MG & $\begin{array}{l}\text { Hospital Escola Da Universidade } \\
\text { Federal Do Triângulo Mineiro }\end{array}$ & - & - & - & - & - & 4 & 4 \\
\hline MG & Biocor Instituto & - & - & - & - & - & 2 & 2 \\
\hline RJ & Hospital São Jose do Avai & - & 2 & - & - & - & - & 2 \\
\hline SP & Hospital Santa Cruz & - & - & - & - & - & 2 & 2 \\
\hline SP & $\begin{array}{l}\text { Hospital dos Fornecedores de } \\
\text { Cana de Piracicaba Djaldrovad }\end{array}$ & 1 & - & - & - & - & - & 1 \\
\hline SP & Hospital Unimed de Sorocaba & 1 & - & - & - & - & - & 1 \\
\hline \multicolumn{2}{|l|}{ Total } & 149 & 811 & 16 & 66 & 29 & 2.715 & 3.786 \\
\hline
\end{tabular}

ES: Espírito Santo; FAEPA: Fundação de Apoio ao Ensino, Pesquisa e Assistência; FMUSP: Faculdade de Medicina da Universidade de São Paulo; HC: Hospital das Clínicas; INCOR: Instituto do Coração; MG: Minas Gerais; RJ: Rio de Janeiro; SP: São Paulo; UF: Unidade da Federação; UFMG: Universidade Federal de Minas Gerais; UNIFESP: Universidade Federal de São Paulo; UNIMED: União dos Médicos; UNICAMP: Universidade Estadual de Campinas.

Tabela 6. Relação de instituições e TOS realizados. Brasil, região Sul, SIH/SUS, 2014. (Ministério da Saúde, 2016a)

\begin{tabular}{|c|c|c|c|c|c|c|c|c|}
\hline UF & Instituição & Coração & Fígado & Pâncreas & Pâncreas/Rim & Pulmão & $\operatorname{Rim}$ & TOTAL \\
\hline RS & $\begin{array}{l}\text { Irmandade Santa Casa de } \\
\text { Misericórdia De Porto Alegre }\end{array}$ & 2 & 63 & - & - & 18 & 244 & 327 \\
\hline SC & Hospital Santa Isabel & 4 & 93 & 2 & 5 & - & 107 & 211 \\
\hline RS & Hospital de Clinicas & 3 & 39 & - & - & 3 & 120 & 165 \\
\hline
\end{tabular}




\begin{tabular}{|c|c|c|c|c|c|c|c|c|}
\hline PR & Hospital Angelina Caron & 11 & 33 & 2 & 19 & - & 90 & 155 \\
\hline SC & Hospital Municipal São Jose & - & 2 & - & - & - & 95 & 97 \\
\hline RS & Hospital São Lucas & - & - & - & - & - & 87 & 87 \\
\hline PR & Hospital Universitário Cajuru & - & - & - & - & - & 56 & 56 \\
\hline PR & $\begin{array}{l}\text { Hospital Universitário } \\
\text { Evangélico de Curitiba }\end{array}$ & - & - & - & - & - & 56 & 56 \\
\hline PR & Hospital São Vicente & - & 29 & - & - & - & 15 & 44 \\
\hline PR & Policlinica Pato Branco Sa & 1 & - & - & - & - & 35 & 36 \\
\hline SC & Imperial Hospital de Caridade & - & - & - & - & - & 31 & 31 \\
\hline PR & Hospital e Maternidade Santa Rita & - & - & - & - & - & 30 & 30 \\
\hline PR & Hospital Infantil Pequeno Príncipe & 4 & 7 & - & - & - & 18 & 29 \\
\hline SC & $\begin{array}{l}\text { Associação Hospitalar Lenoir } \\
\text { Vargas Hospital Regional }\end{array}$ & - & - & - & - & - & 26 & 26 \\
\hline PR & Hospital Santa Casa & 13 & - & - & - & - & 10 & 23 \\
\hline SC & Hospital Governador Celso Ramos & - & - & - & - & - & 23 & 23 \\
\hline PR & Hospital Nossa Senhora das Graças & - & 21 & - & - & - & - & 21 \\
\hline RS & Hospital São Vicente de Paulo & - & 10 & - & - & - & 10 & 20 \\
\hline RS & Hospital Universitário de Santa Maria & - & - & - & - & - & 19 & 19 \\
\hline SC & Hospital Universitário & - & 15 & - & - & - & - & 15 \\
\hline PR & $\begin{array}{l}\text { Cruz Vermelha Brasileira Filial } \\
\text { do Estado do Paraná }\end{array}$ & - & - & - & - & - & 14 & 14 \\
\hline PR & Hospital Salete & - & - & - & - & - & 14 & 14 \\
\hline SC & Hospital Regional Hans Dieter Schmidt & - & - & - & - & - & 14 & 14 \\
\hline PR & Hospital Evangélico de Londrina & - & - & - & - & - & 13 & 13 \\
\hline PR & $\begin{array}{l}\text { Hospital e Maternidade } \\
\text { Maria Auxiliadora }\end{array}$ & - & - & - & - & - & 12 & 12 \\
\hline PR & Santa Casa de Londrina & 3 & - & - & - & - & 8 & 11 \\
\hline PR & Hospital de Clinicas & - & 10 & - & - & - & - & 10 \\
\hline RS & Hospital Bruno Born & - & - & - & - & - & 10 & 10 \\
\hline $\mathrm{RS}$ & Hospital Pompéia & - & - & - & - & - & 7 & 7 \\
\hline RS & Hospital de Caridade de ljui & - & - & - & - & - & 6 & 6 \\
\hline RS & Instituto de Cardiologia & 5 & - & - & - & - & - & 5 \\
\hline RS & $\begin{array}{l}\text { Hospital Universitário São } \\
\text { Francisco de Paula }\end{array}$ & - & - & - & - & - & 2 & 2 \\
\hline & Total & 46 & 322 & 4 & 24 & 21 & 1172 & 1589 \\
\hline
\end{tabular}

PR: Paraná; RS: Rio Grande do Sul; SC: Santa Catarina; UF: Unidade da Federação.

TAP/SUS (para cada órgão sólido) (Ministério da Saúde, 2016b) e quantidade de procedimentos de TOS. O resultado apresentou mais influência dos órgãos sólidos com maior representatividade no valor total: rim e fígado (Tabela 7).

Soma-se a isso o tempo de hospitalização para um paciente transplantado submetido a tratamento preemptivo com GCV intravenoso, considerado como de 21 dias. Já para a profilaxia com VGCV oral, esse valor foi estimado como zero. Como resultados finais, o tempo de internação total por VGCV profilático foi de 11 dias, enquanto o tratamento pre- emptivo foi de 32 dias, ou seja, um incremento de 21 dias de hospitalização.

O tempo médio de hospitalização para qualquer procedimento hospitalar de acordo com o SIH/SUS é de 5,6 dias.

A partir da relação do valor incremental entre a utilização de GCV e VGCV (21 dias) e a média geral de permanência hospitalar no SUS para qualquer procedimento (5,6 dias), pode-se sugerir que 3,75 novas internações por qualquer causa ou finalidade poderiam ocorrer de forma adicional por cada paciente submetido ao VGCV profilático. Em um cenário hipoté- 
Tabela 7. Quantidade de transplantes realizados e média de permanência hospitalar, por órgão. Brasil, SIH/SUS, SIGTAP/SUS, 2014. (Ministério da Saúde, 2016b)

\begin{tabular}{|c|c|c|c|}
\hline Órgão Sólido & Quantidade de transplantes & Código SIGTAP & Média de permanência (dias) \\
\hline Coração & 263 & 05.05.02.004-1 & 16 \\
\hline Fígado & 1.538 & 05.05.02.005-0/05.05.02.006-8 & 14 \\
\hline Pâncreas & 20 & 05.05.02.007-6 & 10 \\
\hline Pâncreas/Rim & 102 & 05.05.02.011-4 & 10 \\
\hline Pulmão & 58 & 05.05.02.008-4/05.05.02.012-2 & 20 \\
\hline $\operatorname{Rim}$ & 4.931 & 05.05.02.009-2/05.05.02.010-6 & 10 \\
\hline \multicolumn{3}{|c|}{ Média de permanência hospitalar (dias) } & 11 \\
\hline
\end{tabular}

SIH/SUS: Sistema de Informações Hospitalares do SUS; SIGTAP: Sistema de Gerenciamento da Tabela de Procedimentos, Medicamentos e OPM; TOS: transplante de órgão sólido.

tico, no qual a falta de leitos representa um fator limitante aos transplantes, 2 novos procedimentos poderiam ser realizados a cada 1 paciente tratado com VGC profilático (Tabela 8).

Ao se aplicar a taxa total de pacientes R- (3,6\%) na população total de transplantados, os pacientes considerados de alto risco D+/R- totalizaram 249 distribuídos em todas as instituições e para todos os tipos de órgãos sólidos analisados. Para as instituições com número significativo de pacientes classificados como população de alto risco (D+/R-) (n maior que um), os resultados para o número de pacientes tratados e as diárias de hospitalização potencialmente evitadas no período estão apresentados na Tabela 9.

De acordo com o panorama encontrado, o número de diárias de hospitalização potencialmente evitadas após adoção de VGCV profilático é de 5.225 em todo o território brasileiro. No Hospital do Rim e Hipertensão, foram estimados 25 pacientes D+/R- e valor resultou em 519 diárias evitadas anualmente. No Hospital das Clínicas da FMUSP, foram estimados 14 pacientes de alto risco, com resultado potencial de redução no tempo de internação de 300 dias anualmente. No caso da Irmandade Santa Casa de Misericórdia de Porto Alegre, foram calculados 12 pacientes de alto risco (D+/R-), um potencial de redução de 247 dias de tempo de internação (Tabela 9).
Em relação ao tipo de órgão sólido, rim e fígado apresentaram maior representatividade em número de transplantes e maiores quantidades de pacientes D+/R-. Desta forma, foram os órgãos com maiores potenciais de desospitalização e consequente oportunidade de novas hospitalizações, sejam elas para realização de outro transplante ou qualquer procedimento hospitalar (Tabela 10).

\section{Discussão}

Este estudo foi desenvolvido com o objetivo de avaliar o panorama atual de TOS no Brasil e entender o impacto da utilização de VGCV profilático na desospitalização.

Ao analisar os dados obtidos, foi possível observar que, independentemente das condições de transplante, tipo de órgão sólido e instituição, a utilização de VGCV profilático tem o potencial de reduzir o tempo de permanência hospitalar em 21 dias. A partir de extrapolações baseadas nos tempos de permanência para transplante ou qualquer procedimento hospitalar, foi percebido o potencial de adicionar procedimentos pela desocupação de leitos.

De um ponto de vista clínico, Hodson et al., 2013, (Hodson et al., 2013) enfatizaram a importância da profilaxia para

Tabela 8. Tempo estimado de permanência hospitalar para VGCV profilático e GCV preemptivo e número potencial de hospitalizações adicionais disponíveis para outros pacientes. (Ministério da Saúde, 2016a)

\begin{tabular}{lccc}
\hline & VGCV Profilático & GCV Preemptivo & Incremental \\
\hline Tempo de hospitalização para transplante (dias) & 11 & 11 & 0 \\
\hline Tempo de hospitalização para CMV (dias) & 0 & 21 & 32 \\
\hline Total de dias de hospitalização & 11 & 21 \\
\hline Tempo de hospitalização para qualquer procedimento hospitalar (dias) & 5,6 & 3,75 & \\
\hline $\begin{array}{l}\text { Número potencial de quaisquer procedimentos hospitalares adicionais } \\
\text { disponibilizados a cada 1 paciente tratado com VGC profilático }\end{array}$ & 2 & \\
\hline $\begin{array}{l}\text { Número potencial de transplantes adicionais por desocupação } \\
\text { de leito a cada 1 paciente tratado com VGC profilático }\end{array}$ & & \\
\hline
\end{tabular}

CMV: citomegalovírus; GCV: ganciclovir; VGCV: valganciclovir. 
Tabela 9. Potencial desospitalização com uso de VGCV profilático, por instituição. (Ministério da Saúde, 2016a)

\begin{tabular}{|c|c|c|}
\hline Instituição & $\begin{array}{l}\text { Número de pacientes } \\
\text { de alto risco (D+/R-) }\end{array}$ & $\begin{array}{c}\text { Diárias de hospitalização } \\
\text { evitadas (dias) }\end{array}$ \\
\hline Hospital Santa Marcelina São Paulo & 1 & 23 \\
\hline Hospital e Maternidade Santa Rita & 1 & 23 \\
\hline Imperial Hospital de Caridade & 1 & 23 \\
\hline Hospital Samaritano & 1 & 24 \\
\hline Hospital das Clinicas Samuel Libanio Pouso Alegre & 1 & 24 \\
\hline Hospital do câncer Antônio Cândido Camargo & 1 & 24 \\
\hline Santa Casa de São Paulo Hospital Central São Paulo & 1 & 25 \\
\hline Hospital Evangélico & 1 & 26 \\
\hline Santa Casa de Misericórdia de São José dos Campos & 1 & 26 \\
\hline Hospital Antonio Targino & 1 & 27 \\
\hline Policlinica Pato Branco Sa & 1 & 27 \\
\hline Hospital de Base do Distrito Federal & 1 & 29 \\
\hline Hospital Marcio Cunha & 1 & 29 \\
\hline Hospital Português & 1 & 29 \\
\hline Santa Casa de Misericórdia de Goiânia & 1 & 29 \\
\hline Hospital São Vicente & 2 & 33 \\
\hline Hospital Evangélico de Vila Velha & 2 & 36 \\
\hline Instituto Dante Pazzanese de Cardiologia São Paulo & 2 & 38 \\
\hline Hospital Ana Nery & 2 & 39 \\
\hline Hospital Universitário Evangélico De Curitiba & 2 & 42 \\
\hline Hospital Universitário Cajuru & 2 & 42 \\
\hline Hospital Geral de Bonsucesso & 2 & 45 \\
\hline Hospital Santa Casa de Montes Claros & 2 & 49 \\
\hline Hospital Universitário Onofre Lopes & 2 & 51 \\
\hline Hospital Jayme da Fonte & 3 & 54 \\
\hline Hospital Adventista Silvestre & 3 & 55 \\
\hline Hospital das Clinicas FAEPA Ribeirão Preto & 3 & 57 \\
\hline Hospital Universitário São Jose & 3 & 58 \\
\hline Santa Casa de Misericórdia de Juiz de Fora & 3 & 60 \\
\hline Hospital São Joaquim Beneficência Portuguesa & 3 & 62 \\
\hline HC da FMUSP Instituto do Coração Incor São Paulo & 3 & 64 \\
\hline Hospital São Lucas & 3 & 66 \\
\hline Hospital Meridional & 3 & 67 \\
\hline Santa Casa de Belo Horizonte & 3 & 68 \\
\hline Hospital de Transplante do Euryclides de Jesus Zerbini & 3 & 70 \\
\hline Hospital das Clinicas da UFMG & 3 & 70 \\
\hline Real Hospital Português & 3 & 70 \\
\hline Hospital Municipal São Jose & 3 & 73 \\
\hline Instituto de Cardiologia do Distrito Federal & 4 & 79 \\
\hline Hospital de Base de São Jose do Rio Preto & 4 & 86 \\
\hline Hospital das Clinicas de Botucatu & 4 & 94 \\
\hline Hospital Bandeirantes & 5 & 104 \\
\hline
\end{tabular}




\begin{tabular}{lll}
\hline Hospital Felicio Rocho & 5 & 115 \\
\hline Hospital Angelina Caron & 6 & 117 \\
\hline Hospital das Clinicas da UNICAMP De Campinas & 6 & 125 \\
\hline Hospital de Clinicas & 6 & 132 \\
\hline Hospital Albert Einstein & 7 & 141 \\
\hline Hospital Geral de Fortaleza & 8 & 153 \\
\hline Hospital Santa Isabel & 8 & 160 \\
\hline Hospital Universitário Walter Cantidio & 8 & 169 \\
\hline IMIP & 10 & 175 \\
\hline Hospital São Francisco de Assis & 12 & 203 \\
\hline Irmandade Santa Casa de Misericórdia de Porto Alegre & 14 & 247 \\
\hline Hospital das Clinicas São Paulo & 25 & 300 \\
\hline Hospital do Rim e Hipertensão & $\mathbf{2 4 9}$ & $\mathbf{5 . 2 2 5}$ \\
\hline Todos & 719 \\
\hline Dt/R-reptos son & \\
\hline
\end{tabular}

D+/R-: receptores soronegativos-CMV cujos doadores são soropositivos-CMV; FAEPA: Fundação de Apoio ao Ensino, Pesquisa e Assistência; FMUSP: Faculdade de Medicina da Universidade de São Paulo; HC: Hospital das Clínicas; IMIP: Instituto de Medicina Integral Professor Fernando Figueira; UNICAMP: Universidade Federal de Campinas; TOS: transplante de órgãos sólidos.

Tabela 10. Resultados da análise em relação ao tipo de órgão sólido. (Ministério da Saúde, 2016a)

\begin{tabular}{lcccc}
\hline Órgão sólido & $\begin{array}{c}\text { Número de pacientes } \\
\text { de alto risco (D+/R-) }\end{array}$ & $\begin{array}{c}\text { Redução de tempo de } \\
\text { hospitalização, em dias }\end{array}$ & $\begin{array}{c}\text { Número potencial de } \\
\text { hospitalizações adicionais } \\
\text { disponibilizadas por ano }\end{array}$ & $\begin{array}{c}\text { Número potencial de } \\
\text { transplantes adicionais } \\
\text { por desocupação de leito }\end{array}$ \\
\hline Coração & 9 & 199 & 36 & 12 \\
\hline Fígado & 55 & 1.163 & 208 & 83 \\
\hline Pâncreas & 1 & 15 & 3 & 2 \\
\hline Pâncreas/Rim & 4 & 77 & 8 & 8 \\
\hline Pulmão & 2 & 44 & 666 & 373 \\
\hline Rim & 178 & 3.728 & & 373 \\
\hline
\end{tabular}

D+/R-: receptores soronegativos-CMV cujos doadores são soropositivos-CMV.

a diminuição do risco de doença pelo CMV e redução das complicações fatais causadas pelo vírus, no contexto do paciente transplantado. Sugere-se ainda que a profilaxia antiviral deveria ser utilizada rotineiramente em receptores de TOS soronegativos-CMV de doadores soropositivos-CMV (Hodson et al., 2013).

Entre as limitações do estudo, destaca-se a impossibilidade de levantamento do número de hospitalizações dedicadas à prevenção da infecção por CMV das bases de dados. A codificação existente não permitiu a identificação de registros correspondentes a este período. Soma-se a isto a carência de dados sobre o desenvolvimento da doença pelo CMV após profilaxia ou tratamento preemptivo, uma vez que as informações presentes na literatura são limitadas, assim como a identificação do período nas bases. Desta forma, essa variável, assim como a eficácia dos tratamentos não foram consideradas nos cálculos (Hodson et al., 2013).

Atualmente, existe outra opção oral além do VGCV, indicado para a profilaxia da doença pelo CMV em pacientes submetidos a TOS, o GCV oral que, apesar de apresentar eficácia semelhante ao VGCV (Paya et al., 2004), não foi considerado na análise. O VGCV foi o medicamento escolhido para a comparação por seu regime posológico simplificado. A dosagem do GCV oral consiste em 4 cápsulas ingeridas durante 3 períodos diários (Blau Farmacêutica S.A., 2014), enquanto - VGCV necessita de apenas 2 comprimidos administrados uma vez ao dia (Roche, 2014).

De acordo com o estudo desenvolvido, VGCV é uma opção vantajosa para potencializar o número de leitos livres, essencialmente pela falta de necessidade de hospitalizar o paciente durante a administração do medicamento, ao contrário do que ocorre com o GCV intravenoso em tratamento preemptivo, situação em que os pacientes necessitam de duas infusões diárias por um período mínimo de 21 dias, sob supervisão e monitoramento da viremia.

Considerada uma realidade de recursos escassos, principalmente no âmbito de saúde universal, e de intenso trabalho com o programa de transplantes nacional, aliada à 
necessidade de desospitalização para a gestão eficiente de recursos, a liberação de leitos pode vir a favorecer a utilização dos recursos hospitalares para um número maior de pacientes.

\section{Conclusão}

As informações obtidas nesse estudo permitem concluir que a utilização de VGCV oral em regime profilático é potencialmente capaz de promover a desospitalização, permitindo maior comodidade ao paciente transplantado e favorecendo a utilização de recursos pela instituição.

\section{Referências bibliográficas}

Alexopoulos SP, Lindberg L, et al. Cytomegalovirus prophylaxis in solid organ transplantation. Curr Med Chem. 2012;19(35):5957-63.

Associação Brasileira de Transplante de Órgãos (ABTO). Dimensionamento dos transplantes no Brasil e em cada estado (2007-2014). Registro Brasileiro de Transplantes. São Paulo: ABTO; 2014. 98 p.

Blau Farmacêutica S.A. Ganvirax ${ }^{\circledast}$ (ganciclovir) [Bula]. 2014. 6 p.

Bordonaro S, Raiti F, et al. Active home-based cancer treatment. J Multidiscip Healthc. 2012;5:137-43.

Bruminhent J, Razonable RR. Management of cytomegalovirus infection and disease in liver transplant recipients. World J Hepatol. 2014;6(6):370-83.

Giakoustidis D, Antoniadis A, et al. Prevalence and clinical impact of cytomegalovirus infection and disease in renal transplantation: ten years of experience in a single center. Transplant Proc. 2012;44(9):2715-7.

Harvala $\mathrm{H}$, Stewart $\mathrm{C}$, et al. High risk of cytomegalovirus infection following solid organ transplantation despite prophylactic therapy. J Med Virol. 2013:85:893-8
Hodson E, Ladhani M, et al. Antiviral medications for preventing cytomegalovirus disease in solid organ transplant recipients (Review). Cochrane database Syst Rev. 2013;(2).

Hospital Israelita Albert Einstein. Diretrizes Assistenciais Protocolo de Tratamento de Infecção pelo Citomegalovírus ( CMV ). 2011. p. 1-4.

Hospital Israelita Albert Einstein. Diretrizes Assistenciais Protocolo de Tratamento de Infecção pelo Citomegalovírus ( CMV ). 2012. p. 1-9.

Junqueira M, Sancho T, et al. Citomegalovírus: revisão dos aspectos epidemiológicos, clínicos, diagnósticos e de tratamento. Newslab. 2008;86:88-104.

Marinho A. Um estudo sobre as filas para transplantes no Sistema Único de Saúde brasileiro. Cad Saúde Pública. 2006;22(10):2229-39.

Ministério da Saúde (Brasil). Secretaria de Atenção à Saúde (SAS). Departamento de regulação e avaliação e controle (DRAC). [site na Internet] Sistema de Informações Hospitalares do SUS - SIH/SUS. 2016a [atualizado 2016; citado 2016 fev]. Disponível em: http://ces.ibge. gov.br/base-de-dados/metadados/ministerio-da-saude/sistema-deinformacoes-hospitalares-do-sus-sih-sus.htm

Ministério da Saúde (Brasil). Departamento de Informática do SUS (DATASUS). [site na Internet] Sistema de Gerenciamento da Tabela de Procedimentos, Medicamentos e OPM do SUS (SIGTAP). 2016b [atualizado 2016; citado 2016 fev]. Disponível em: http://sigtap.datasus.gov.br

Paya C, Humar A, et al. Efficacy and safety of valganciclovir vs. oral ganciclovir for prevention of cytomegalovirus disease in solid organ transplant recipients. Am J Transplant. 2004;4:611-20.

Produtos Roche Químicos e Farmacêuticos S.A. Valcyte ${ }^{\oplus}$ (cloridato de valganciclovir) [Bula]. 2014. 18 p.

Razonable RR. Cytomegalovirus Infection After Liver Transplantation. Liver Transplant. 2010;16(10):45-53.

Roman A, Manito N, et al. The impact of the prevention strategies on the indirect effects of CMV infection in solid organ transplant recipients. Transplant Rev. 2014;28(2):84-91.

Souza MA, Passos AM, et al. Seroprevalence of cytomegalovirus antibodies in blood donors in southern, Brazil. Rev Soc Bras Med Trop. 2010;43(4):359-61. 